\title{
EXERCÍCIOS LIVRES VERSUS DIRECIONADOS NAS COMPLICAÇÕES PÓS-OPERATÓRIAS DE CÂNCER DE MAMA
}

Laura Ferreira de Rezende, Patricia Odila Beletti, Ricardo Laier franco, Sirle Siani Moraes, Maria Salete Costa Gurgel*

Trabalho realizado no Serviço de Fisioterapia, Departamento de Tocoginecologia, Universidade Estadual de Campinas (CAISM-UNICAMP)

\author{
*Correspondência: \\ Departamento de \\ Tocoginecologia da FCM - \\ UNICAMP \\ Rua Alexander Flemming, 101 \\ Cidade Universitária \\ Zeferino Vaz, Campinas, SP, \\ Brasil, Cep 13081-970, \\ Fone/Fax: (19) 3788-9516/ \\ $3788-9401$
}

\begin{abstract}
RESUMO
OBjetivo. Avaliar a associação entre o tipo dos exerćícios fisioterápicos com a incidência de complicações pós-operatórias em mulheres submetidas à mastectomia radical ou quadrantectomia com linfadenectomia axilar.

Métodos. Ensaio clínico randomizado com 60 mulheres. 0 grupo direcionado fez exercícios seguindo um protocolo preestabelecido de 19 exercícios $(n=30)$ e o grupo livre fez exercícios sem seqüência e número de repetições preestabelecidos $(n=30)$. Realizou-se uma avaliação inicial da paciente na fase pré-operatória e reavaliações nos $14^{\circ}$, $28^{\circ}$ e 42 - dias pós-operatório, analisando-se como volume total de secreção drenada, tempo total de permanência do dreno, incidência de seroma e de deiscência da ferida cirúrgica e circunferência do membro superior ipsolateral.

Resultados. 0 grupo direcionado permaneceu, em média, $12,17 \pm 2,96$ dias com o dreno, enquanto o grupo livre 11,96 \pm 2,32 dias, não havendo diferença significativa entre os grupos. A quantidade de secreção drenada durante a permanência do dreno também se mostrou semelhante entre os grupos, sendo em média de $|308,7| \pm 562,6 \mathrm{ml}$ no grupo direcionado e |39|,62 — 644,65ml no grupo de exercícios livres. As incidências de seroma e de deiscência da ferida cirúrgica não se mostraram influenciadas pelo tipo de exercício em nenhum dos momentos avaliados. No grupo direcionado, 7,4\% e 3,4\% das mulheres apresentaram seroma nos 280 e $42 \circ$ dias, respectivamente, enquanto $3,6 \%$ e $3,6 \%$ do grupo livre, respectivamente, nos mesmos momentos de avaliação. A incidência de deiscência da ferida cirúrgica também foi semelhante nos dois grupos, sendo que no grupo direcionado foi de $20 \%$ no 140 dia, $31 \%$ no 280 dia e $10,3 \%$ no 420 dia de pósoperatório. No grupo livre a incidência foi de $23,3 \%$ no 14 - dia, 33,3\% no 28 dia e 22,2\% no 420 dia de pós-operatório. A circunferência do membro superior no grupo direcionado não apresentou diferença significativa em nenhum dos momentos avaliados.
\end{abstract}

Conclusão. As complicações não se apresentaram influenciadas pela forma de aplicação dos exercícios.

UnIteRMos: Linfadenectomia axilar. Câncer de mama. Fisioterapia. Reabilitação.

\section{INTRODUÇÃO}

O carcinoma mamário invasor é a segunda neoplasia mais incidente na população feminina do Brasil, com 41.610 casos novos diagnosticados e 9.335 óbitos em 2003!. A imensa maioria das pacientes submete-se a abordagem cirúrgica da axila com finalidade prognostica e o pós-operatório pode levar a complicações como seroma, deiscência da ferida cirúrgica e linfedema precoce. Considerando a expectativa de sobrevida de 17,5 anos para essas mulheres e que o método gold-stantard para estadiamento da axila é o esvaziamento axilar, tornou-se fundamental o aprimoramento das técnicas de reabilitação para proporcionar uma adequada qualidade de vida física e mental ${ }^{2}$.

Os exercícios realizados nos programas de reabilitação física no pós-operatório de câncer de mama não seguem um guideline. Muitas propostas de reabilitação foram desenvolvidas para minimizar as complicações pós-operatórias, como o volume de secreção drenada, a incidência de seroma, de deiscência da ferida cirúrgica e, a longo prazo, o desenvolvimento de linfedema crônico.

Alguns estudos discutem a associação entre a realizaçãa dos exercícios com as possíveis complicações do pós-operatório; entretanto na literatura há descrições sucintas e particularizadas sobre a maneira de realização dos exercícios.
A fisioterapia precoce tem como objetivos prevenir complicações, promover adequada recuperação funcional e, conseqüentemente, propiciar melhor qualidade de vida às mulheres submetidas à cirurgia para tratamento de câncer de mama $a^{3,4}$. Entretanto, questiona-se qual a melhor maneira de realizar esses exercícios e qual a sua influência nas complicações pós-operatórias.

Os programas de reabilitação no pós-cirúrgico das pacientes submetidas à mastectomia ou a tratamento conservador com dissecção axilar são parcialmente descritos na literatura do ponto de vista da especificação dos exercícios realizados. Existem programas estruturados em contrações isométricas da musculatura do ombro, braço e mão, nos quais a paciente é instruída a levantar, com as mãos unidas, em flexão, abdução e rotação do ombro até o limite de dor ${ }^{5}$; em outros em que a paciente é estimulada a realizar exercícios ativo-livres em todos os movimentos fisiológicos do ombro6.

Há, também, terapias em que são indicados os exercícios como subir com os dedos pela parede até o limite máximo de flexão e abdução, pentear os cabelos, fazer roda de ombro e rotação do braço, entre outros ${ }^{7}$. Existem propostas baseadas em alongamento e fortalecimento, com exercícios rítmicos de cabeça, pescoço, tronco, membros superiores e inferiores. ${ }^{8}$. Outros 
Rezende LF ET AL.

programas consistem de exercícios pendulares, exercícios de escalada do braço na parede e polias?

Wingate ${ }^{3}$ descreve o tratamento incluindo exercícios ativoassistidos progredindo para exercícios ativo-resistidos, facilitação neuromuscular proprioceptiva e atividades funcionais, além de orientações para casa. Molinaro et al. ${ }^{10}$ defendem um protocolo de exercício baseado em movimentos naturais, acompanhados de música, para desenvolver flexibilidade, coordenação e amplitude de movimento do ombro.

No tratamento de câncer de mama existe um certo consenso no que diz respeito a cirurgia, quimioterapia, radioterapia e hormonioterapia; no entanto, na reabilitação física não existem trials comparando e tentando padronizar os programas de exercícios. Diante de várias propostas, têm-se a necessidade de homogeneização dos protocolos, para que sua reprodutibilidade seja possível, dentro de um serviço com vários fisioterapeutas ou em outros serviços, para que 0 atendimento possa ser otimizado, além de permitir a comparação dos resultados obtidos.

O objetivo deste estudo é avaliar a associação entre o tipo dos exercícios fisioterápicos realizados - direcionados ou livres, com a incidência de complicações em mulheres submetidas a mastectomia radical ou quadrantectomia com linfadenectomia axilar na rotina do serviço de fisioterapia CAISM/UNICAMP.

\section{Métodos \\ Pacientes}

Foi realizado um ensaio clínico controlado randomizado. Foram selecionadas, para participar desse estudo, mulheres com câncer de mama, submetidas a mastectomia radical ou quadrantectomia com linfadenectomia axilar, internadas na Enfermaria de Oncologia do CAISM-UNICAMP, no período de 23/03/2003 a 13/07/2003. Para serem incluídas no estudo, as pacientes precisavam estar realizando a primeira cirurgia por carcinoma invasivo de mama ipsilateral. Foram excluídas as mulheres que realizaram reconstrução mamária imediata ou cirurgia bilateral, que apresentaram diferença na circunferência dos membros superiores maior que $2 \mathrm{~cm}$ prévia à cirurgia, que apresentaram limitação de movimento no membro ipsilateral à cirurgia avaliado como diferença maior que 20 graus na flexão e abdução prévia à cirurgia, e aquelas com incapacidade de compreender os exercícios propostos.

A técnica de fisioterapia utilizada foi a cinesioterapia visando exercícios de flexão, extensão, abdução, adução, rotação interna e rotação externa dos membros superiores, isolados ou combinados. O grupo direcionado realizou a fisioterapia seguindo um protocolo de 19 exercícios (Quadro I).

O grupo livre realizou os exercícios seguindo os movimentos fisiológicos da biomecânica do ombro, sem uma sequeência e número de repetições previamente definidos, sendo os exercícios realizados acompanhando o ritmo da música ${ }^{10}$. Três exercícios foram iniciados no primeiro dia após a cirurgia em ambos os grupos. Todas as pacientes foram orientadas a manter atividade livre com o membro acometido em suas atividades diárias. Após 48 horas da cirurgia, os exercícios passaram a ser realizados no ambulatório de fisioterapia do CAISM-

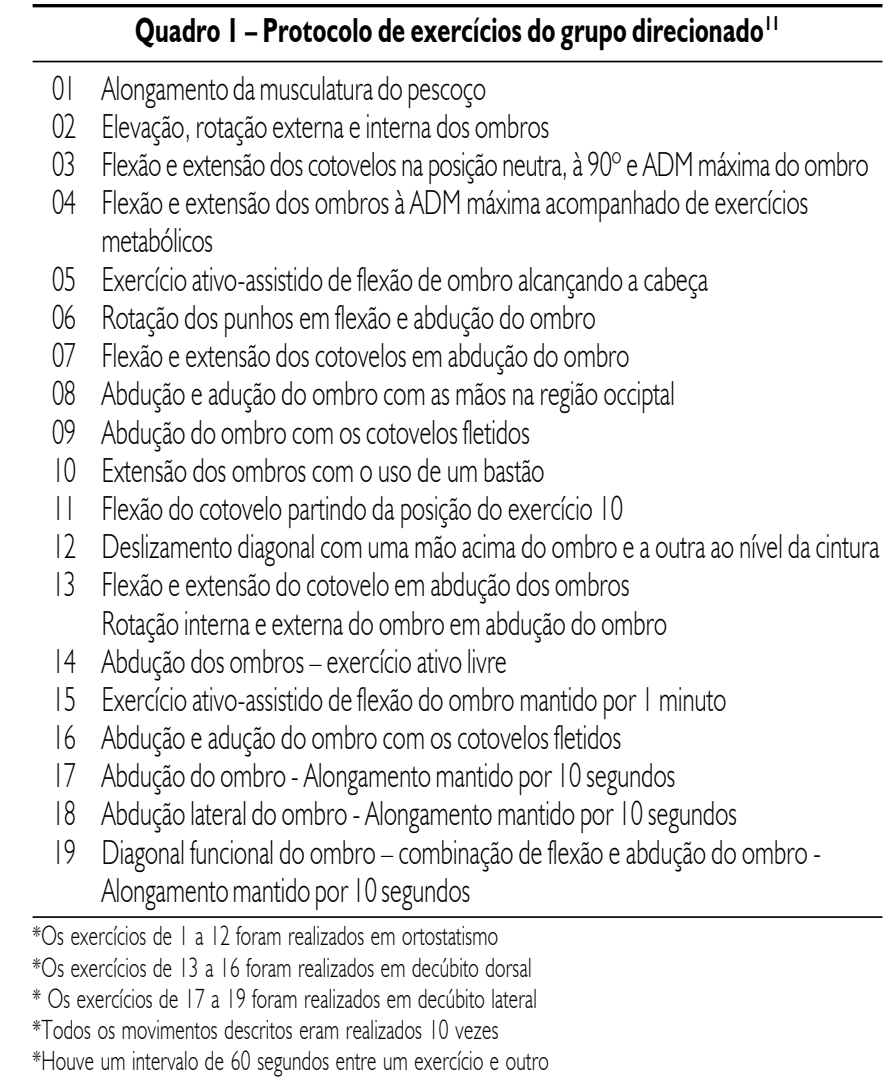

UNICAMP, com sessões de 40 minutos, freqüência de três vezes por semana, por um período de 42 dias após a cirurgia. Os exercícios foram aplicados em grupos de 5 a 20 mulheres, e ministrados por uma equipe constituída por cinco fisioterapeutas e 10 especializandas.

\section{Testes avaliatórios}

A cirtometria foi realizada para medir a circunferência do membro superior em centímetros ${ }^{12}$. O instrumento utilizado foi uma fita métrica flexível (marca Hoachstmass), realizada a 7,5 centímetros acima e abaixo da prega do cotovelo, na articulação metacarpofalangiana e no processo estilóide da una". Para realizar a cirtometria, o sujeito permaneceu com a região a ser avaliada despida.

O volume de secreção drenada foi mensurado pela própria paciente, que recebeu uma mamadeira plástica graduada no pré-operatório. Nos dois primeiros dias, o volume foi medido pelo serviço de enfermagem do CAISM/UNICAMP. Nos demais dias, a paciente, que recebeu alta, foi orientada a esvaziar uma vez ao dia o dreno e a medir, na mamadeira, o volume de secreção drenada e anotar em um formulário próprio.

O volume e a incidência de seroma foi medido pelo serviço de enfermagem. A presença ou não de deiscência, bem como a cirtometria do membro superior, foi avaliada no serviço de fisioterapia.

\section{Implicações éticas}

A realização deste estudo baseou-se na Declaração de Helsinque ${ }^{13}$ e na Resolução 196/96 14 . O projeto inicial foi aprovado pela 
Comissão de Pesquisa do Departamento de Tocoginecologia e pelo Comitê de Ética em Pesquisa da Faculdade de Ciências Médicas/UNICAMP.

\section{Métodos estatísticos}

Os dados foram avaliados descritivamente através de média, desvio, no caso de variáveis contínuas, através dos testes $T$ de Student e Mann-Whitney; pelo cálculo de frequêencias e percentuais para as variáveis categóricas, através dos testes Qui-quadrado ou Exato de Fisher. A MANOVA foi o principal instrumento para a avaliação dos dados, tendo sido utilizado na verificação de tendência no tempo, comparação dos tempos em cada grupo e para testar o efeito de algumas variáveis de controle.

\section{Resultados}

Foram acompanhadas 60 mulheres no pós-operatório de câncer de mama, sendo 30 no grupo direcionado e 30 no grupo livre (Tabela I). Não houve diferença significativa entre os grupos em relação às características individuais e clinico-cirúrgicas das pacientes.

A incidência de infecçãa foi semelhante nos dois grupos, sendo de $20 \%$ no 14 o dia de pós-operatório, de $18,6 \%$ no 28 - dia e de $10,5 \%$ no 42 - dia de pós-operatório. Vale ressaltar que duas mulheres apresentaram infecção no $14^{\circ}$ e no $28^{\circ}$ dia, duas no $28^{\circ}$ e $42^{\circ}$ dia, uma no $144^{\circ}$ e $42^{\circ}$ dia, e duas nos três momentos de avaliação.

Os grupos foram homogêneos em relação ao número de sessões de fisioterapia realizadas, tendo sido realizadas $13,83 \pm 3,05$ sessões no grupo direcionado e 13,19 1 1,9 sessões no grupo livre.

\section{Complicações pós-operatórias}

O grupo direcionado permaneceu, em média, $12,17 \pm 2,96$ dias com o dreno, enquanto o grupo livre $11,96 \pm 2,32$ dias, não havendo diferença significativa entre os grupos.

A quantidade de secreção drenada durante a permanência do dreno também mostrou-se semelhante entre os grupos, sendo em média de $|308,7| \pm 562,6 \mathrm{ml}$ no grupo direcionado e 1391,62 $\pm 644,65 \mathrm{ml}$ no grupo de exercícios livres.

A circunferência do membro superior no grupo direcionado não apresentou diferença significativa em nenhum dos momentos avaliados. A diferença estatística observada entre as médias das circunferências da mão e do braço medidas no pré-operatório e comparadas às observadas no 420 dia de pós-operatório não traduzem diferenças clínicas. No grupo livre pudemos observar uma diferença estatisticamente significativa na circunferência do punho e do braço, que não também corresponde a diferença clínica significativa (Figuras l-4).

\section{Fatores que influenciam o volume do braço}

Foi realizada uma análise multivariada para se observar a influência do estadiamento cirúrgico, tipo de cirurgia, tempo de permanência do dreno, volume de secreção drenada e complicações como seroma, deiscência e infecção da ferida cirúrgica sobre as médias de circunferência do membro superior (Tabela 2). Foram observadas significância estatística do tempo de retirada do dreno, e de complicações como a presença de seroma e de infecção nas médias de circunferência da mão, punho, antebraço e braço. Não foi observada associação com
Tabela I - Variáveis de controle segundo o grupo de exercícios

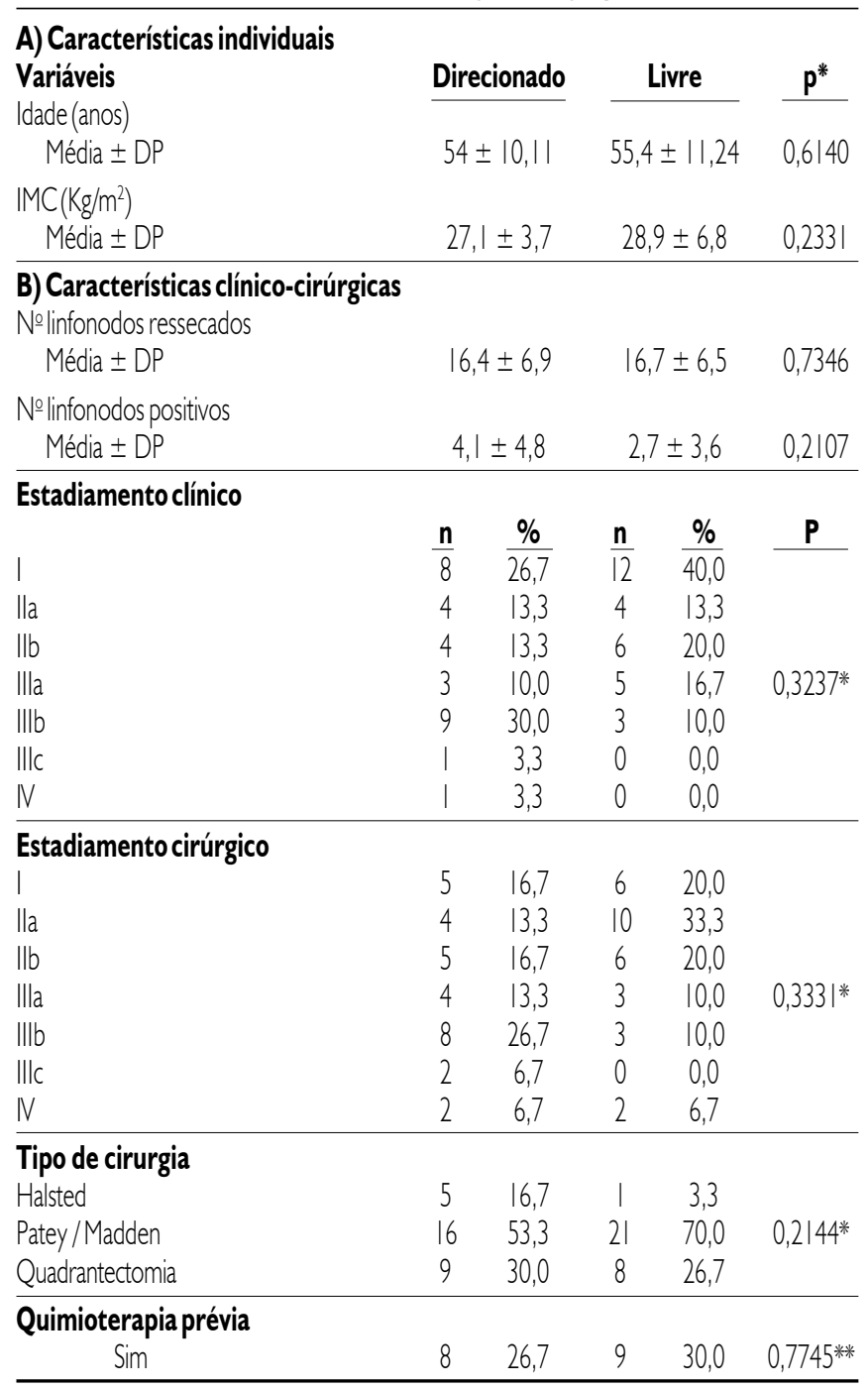

\section{Figura I - Distribuição das médias da circunferência da mão ao longo do tempo de estudo e segundo o grupo de exercícios}

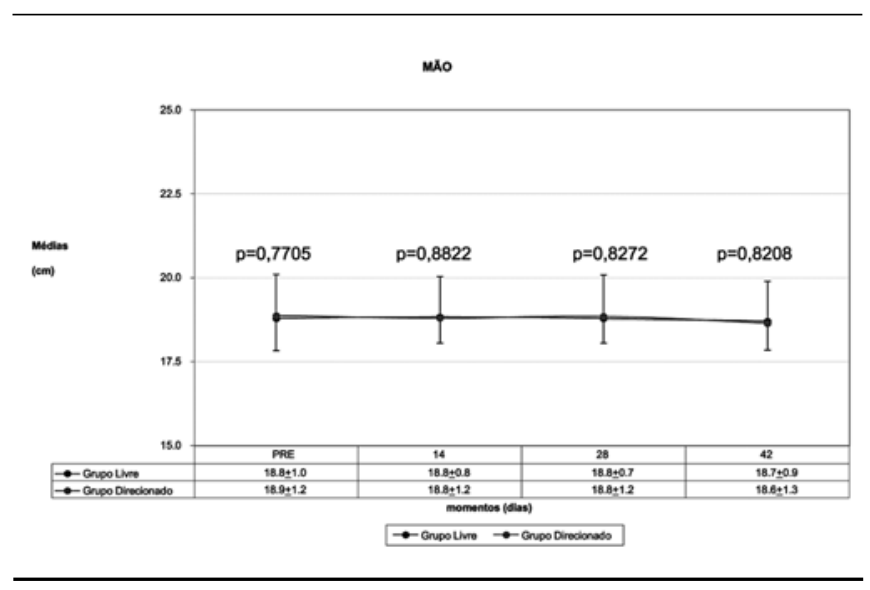




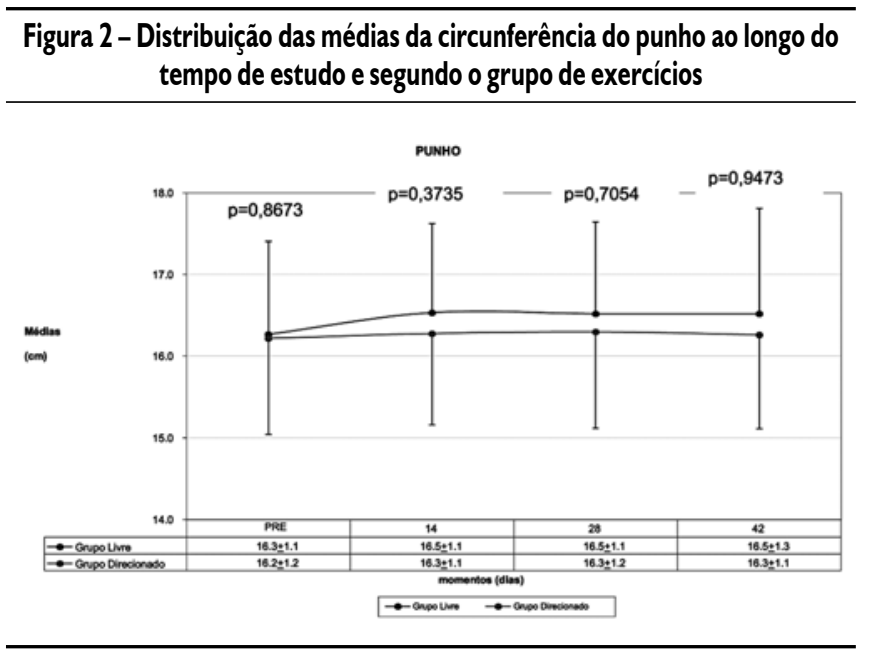

Figura 3 - Distribuição das médias da circunferência do antebraço ao longo do tempo de estudo e segundo o grupo de exercícios

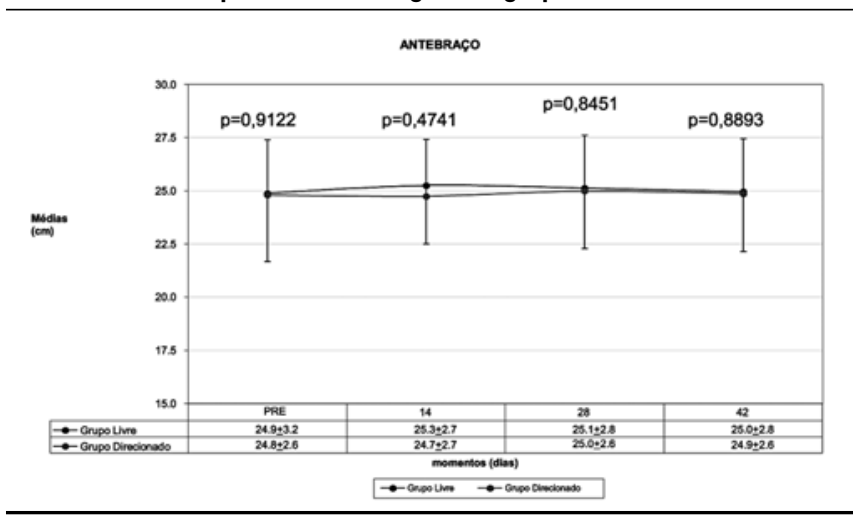

Tabela 2 - Incidência de seroma e deiscência nos momentos avaliados segundo os grupos de exercícios

\begin{tabular}{|c|c|c|c|c|c|}
\hline \multirow[t]{2}{*}{ Dia de pós-operatório } & \multicolumn{4}{|c|}{ Direcionado Livre } & \multirow[t]{2}{*}{$\mathrm{p}^{*}$} \\
\hline & $\overline{\mathbf{N}}$ & $\%$ & $n$ & $\%$ & \\
\hline \multicolumn{6}{|l|}{ A) Incidência de seroma } \\
\hline 14ㅇ dia & & & & & \\
\hline Presente & 0 & 0 & 2 & 7,1 & 0.2228 \\
\hline 280 dia & & & & & \\
\hline Presente & 2 & 7,4 & । & 3,6 & 0.6110 \\
\hline $42 \div 0 \mathrm{dia}$ & & & & & \\
\hline Presente & । & 3,4 & । & 3,6 & 1.000 \\
\hline
\end{tabular}

B) Incidência de deiscência da ferida cirúrgica

\begin{tabular}{lccccc}
$\mid 4^{\circ}$ dia & & & & & \\
Presente & 6 & 20 & 7 & 23,3 & 0.7540 \\
$\begin{array}{l}28^{\circ} \text { dia } \\
\text { Presente } \\
42^{\circ} \text { dia }\end{array}$ & 9 & 31 & 10 & 33,3 & 0.8502 \\
Presente & 3 & 10,3 & 6 & 22,2 & 0.2884 \\
\hline
\end{tabular}

Figura 4 - Distribuição das médias da circunferência do braço ao longo do tempo de estudo e segundo o grupo de exercícios

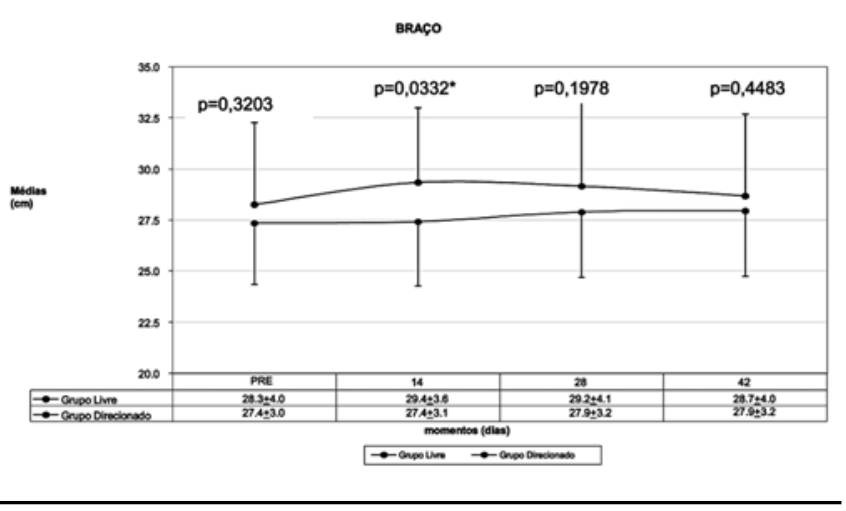

estadiamentos clínico e cirúrgico, tipo de cirurgia, volume de secreção drenada e deiscência da ferida cirúrgica. Provavelmente, haja interferência das variáveis avaliadas em todos os movimentos, mas o tamanho de amostra não permitiu o esclarecimento definitivo.

\section{Discussão}

Este ensaio clínico randomizado comparou duas maneiras de realização dos exercícios do ombro no pós-operatório imediato de câncer de mama, com o intuito de observar se a forma de realização e a escolha criteriosa dos movimentos a serem realizados influenciam nas complicações pós-mastectomia radical ou quadrantectomia com linfadenectomia axilar.

Os resultados demonstraram que não se evidenciou associação entre o modo de realização dos exercícios e o tempo de permanência do dreno, o volume de secreção drenada, a incidência de seroma, deiscência e linfedema precoce.

Encontramos vários estudos que apresentam variações importantes na forma de aplicação dos exercícios. Existem propostas baseadas em exercícios ativo-livres ${ }^{6,7}$ e/ou ativo-assistidos pelo outro membro ${ }^{5}$ ou por polias ${ }^{9}$. Outros programas sugerem alongamentos ${ }^{8}$ e reabilitação funcional $^{3}$. Entretanto, não são descritos os protocolos completos de uma sessão de fisioterapia, ou seja, quais exercícios foram realizados, a duração e o número de repetições. Dessa forma, ao comparar os resultados obtidos em diversos estudos, surge a questão metodológica dos exercícios como um fator que dificulta a análise e a reprodutibilidade em outros serviços.

Procedendo-se à revisão da literatura, pode-se perceber que a questão da predisposição à formação de seroma quando os exercícios são realizados no pós-operatório imediato ainda é discutível. Existem estudos que sugerem que o início precoce dos exercícios de ombro levaria a um aumento na incidência de seroma ${ }^{15,16,17,18}$ e outros, mostram que, além do início tardio levar a uma menor formação de seroma, não há prejuízo à amplitude de movimento articular a longo prazo $6,9,17,18$. Por outro lado, podem ser encontrados estudos que não observaram a associação entre início precoce dos exercícios e a maior incidência de seroma ${ }^{3,4,5}$, sugerindo as vantagens do início precoce dos exercícios na recuperação física da mulher. 
Estudos comparando o volume drenado em grupos que iniciaram os exercícios precocemente e grupos que iniciaram exercícios tardios observaram os seguintes valores: Dawson et al..$^{16}$, em um estudo prospectivo randomizado, com 100 mulheres pós-mastectomia radical modificada, observaram uma drenagem 15\% maior no grupo que iniciou os exercícios no primeiro dia após a cirurgia (322 $\pm 169 \mathrm{ml}$ ) para $379 \pm 208 \mathrm{ml}$ no grupo que iniciou os exercícios no quinto dia de pós-operatório, sendo esta diferença significante estatisticamente. Jansen et al. ${ }^{17}$, em estudo também prospectivo randomizado, com 163 mulheres pós-mastectomia radical modificada, encontraram 14\% menos de drenagem no grupo de início tardio - oitavo dia, (600 4436 $\mathrm{ml}$ para $70 \mathrm{l} \pm 398 \mathrm{ml}$ ), mas esta diferença não foi significante.

Chen e Chen ${ }^{9}$ acompanharam 344 mulheres, randomizadas em 3 grupos - de início no $3^{\circ}$ dia de pós-operatório, no $6^{\circ}$ dia e após a retirada do dreno. A média de drenagem na fossa axilar foi significativamente menor $(p=0,032)$ no grupo que iniciou os exercícios após a retirada do dreno (485 ml, $568 \mathrm{ml}$ e $559 \mathrm{ml}$, respectivamente), sem apresentar diferença, entretanto, na média de drenagem da parede do tórax e no número de aspirações de seroma.

O tempo médio de permanência do dreno foi semelhante nos grupos e dentro da variabilidade esperada segundo os dados da literatura. $\bigcirc$ volume médio de secreção drenada nesse período, medido diariamente pelas mulheres, também não apresentou diferença significativa entre os grupos. Comparando com outros estudos que também iniciaram os exercícios no pós-operatório imediato, este apresentou valores maiores, tendo Van Der Horst et al. ${ }^{5}$ encontrado 935 (210-3.840)ml em média e Jansen et al. ${ }^{17}, 701$, 4 + 397,7ml. Por outro lado, se comparado a estudos nos quais os exercícios foram iniciados mais tardiamente, essa diferença torna-se mais importante. Van Der Horst et al. ${ }^{5}$ relatam o volume médio de 817 (70-2.600)ml e Jansen et al. ${ }^{17} 600,1 \pm 435,6 \mathrm{ml}$ com exercícios iniciados no oitavo dia de pós-operatório.

Apesar desse volume de secreção aumentado, a incidência total de seroma foi de 5,5\% no $28^{\circ}$ dia e 3,5\% no 42 dia de pós-operatório, não tendo apresentado diferença entre os grupos de exercícios. Não se pode afirmar, entretanto, observando esses resultados, que o aumento da drenagem seja responsabilidade do exercício precoce ${ }^{\prime \prime}$. Fatores como o tempo de duração da cirurgia, estadiamento da neoplasia, índice de massa corpórea, idade da paciente, técnica cirúrgica, infecção, além do esvaziamento axilar, podem estar influenciando nessa drenagem $7,6,9,19$.

Aderências são esperadas com maior rapidez em pacientes imobilizadas assim como o afastamento das bordas cirúrgicas em mulheres submetidas ao exercício livre ${ }^{5,15}$. Entretanto, existem estudos que não mostram a associação entre deiscência e o exercício precoce sem restrição de amplitude"l.

A incidência de deiscência da ferida cirúrgica também não apresentou diferença entre os grupos direcionados e livres. Esses valores são menores que os encontrados por Silva et al. "' - 47\% - em estudo realizado no mesmo serviço, mas maiores que os índices obtidos por Jansen et al. ${ }^{17}-15,6 \%$. Muitos são os fatores envolvidos na ocorrência da deiscência como a idade e quadro clínico da paciente, técnica cirúrgica, experiência do cirurgião, número de dias de internação, extensão da dissecção axilar, envolvimento dos linfonodos, presença de dreno, volume de perda sanguínea e índice de massa corpórea ${ }^{6}$, não podendo, portanto, sugerir que os exercícios de início precoce seriam os responsáveis pela maior incidência de deiscência pós-operatória.

Houve diferença significativa na circunferência do braço homolateral à cirurgia no |40 dia de pós-operatório no grupo livre, que não se manteve nos outros momentos de avaliação. Essa diferença numérica não traduz alteração clínica evidente. Não foram encontradas diferenças de circunferência no grupo direcionado e no antebraço, punho e mão do grupo livre. Sabe-se que o linfedema crônico é uma complicação tardia do câncer de mama e não são esperadas alterações precoces de circunferência.

Entretanto, fatores como a idade, número de linfonodos dissecados, número de linfonodos positivos, índice de massa corpórea, extensão cirúrgica e radioterapia influenciam no desenvolvimento do linfedema ${ }^{12}$. Faz parte da rotina do serviço de fisioterapia do CAISM/ Unicamp a distribuição de manuais no pós-operatório contendo informações preventivas do linfedema, mas talvez a orientação precoce de medidas físicas, tais como a automassagem, minimizasse essa complicação no futuro.

O tamanho amostral não permitiu que a análise multivariada esclarecesse definitivamente a influência ou não das variáveis como o estadiamento cirúrgico, tipo de cirurgia, tempo de permanência do dreno, volume de secreção drenada e complicações como seroma, deiscência e infecção da ferida cirúrgica nas médias de circunferência do membro superior ao longo dos 42 dias. No entanto, as observações de significância estatística em determinadas medidas de determinado grupo de estudo reforçam em muito a provável interferência dessa variável de controle no resultado final, sendo, assim, fatores importantes a serem levados em conta no programa de reabilitação.

\section{ConClusão}

Novos estudos serão necessários sobre a técnica de aplicação dos exercícios, bem como a padronização metodológica sobre os exercícios nos serviços de reabilitação e na literatura de maneira geral, uma vez que foi possível perceber a sua influência nos resultados obtidos. O importante é adaptar a reabilitação física para que se possa alcançar resultados mais eficientes para as mulheres no pós-operatório de câncer de mama.

Conflito de interesse: não há.

\section{SUMMARY}

RANDOM CLINICAL COMPARATIVE TRIAL BETWEeN FREE AND DIRECTED EXERCISE IN POST-OPERATIVE COMPLICATIONS OF BREAST CANCER

INTRODUCTION. The objective of this study was to evaluate the association among physiotherapy exercises performed - directed or freein case of post-operative complication in women who underwent radical mastectomy or quadrantectomy with axillary dissection.

METHODS. Sixty women were randomized. The directed group performed physiotherapy following a regimen of 19 exercises. The free group performed the exercises following the biomechanical physiological movements of the shoulder without a previously defined sequence or number of repetitions, exercises were done to the rhythm of music. 
REZENDE LF ET AL.

RESULTS. In the average, the directed group remained $12.17+2.96$ days with the drain, while the free group remained I I.96 + 2.32 days, with no significant difference between groups. The amount of secretion drained during the permanence of the drain also revealed resemblance between groups, with an average of $1308.71+562.6 \mathrm{~m} /$ in the directed group and of $1391.62+644.65 \mathrm{ml}$ in the group of free exercises. Incidences of seroma and dehiscence of the surgical wound did not seem to be influenced by the type of exercise at any of the times evaluated. In the directed group, $7.4 \%$ and $3.4 \%$ of the women presented with seroma on the $28^{\text {th }}$ and $42^{\text {nd }}$ days, respectively, while at the same moments of evaluation the free group presented respectively. 3.6\% and 3.6\%. Incidence of dehiscence of the surgical wound also was similar in the two groups, in the directed group it was of $20 \%$ on the $14^{\text {th }}$ day, $31 \%$ on the $28^{\text {th }}$ day and $10.3 \%$ on the $42^{\text {nd }}$ day of postoperative. In the free group the incidence was of $23.3 \%$ on the $14^{\text {th }}$ day, of $33.3 \%$ on the $28^{\text {in }}$ day and of $22.2 \%$ on the $42^{\text {nd }}$ day of postoperative. The statistical difference observed in the averages of the hand and arm circumferences does not translate into clinical differences.

CONCLUSION. The complications were not influenced $f$ by the physiotherapy exercises performed. [Rev Assoc Med Bras 2006; 52(I): 37-42]

KEY wORDS: Axillary dissection. Breast cancer. Physiotherapy. Rehabilitation.

\section{REFERÊNCIAS}

I. Brasil. Ministério Da Saúde. INCA Instituto Nacional de Câncer 2002 [on line] [citado I 5 nov 2003]. Disponível em: http://www.inca.gov.br.

2. Holmes CE, Muss HB. Diagnosis and treatment of breast cancer in the eldery. CA Cancer J Clin 2003;53:227-44.

3. Wingate L. Efficacy of phisical therapy for patients who have undergone mastectomies. Phys Ther 1985;65:896-900.

4. Wingate L, Croghan I, Natarajan N, MichalekAM, Jordan C. Rehabilitation of the mastectomy patient: a randomized, blind, prospective study. Arch Phys Med Rehabil 1989;70:21-4.

5. Van Der Horst ChMAM, Kenter JAL, De Jong MT, Keeman JN. Shoulder function following early mobilization of the shoulder after mastectomy and axillary dissection. Neth J Surg 1985;37: 105-8.
6. Schultz I, Barholm M, Grondal S. Delayed shoulder exercises in reducing seroma frequency after modified radical mastectomy: a prospective randomized study. Ann Surg Oncol 1997;4:293-7.

7. Aitken DR, Minton JP. Complications associated with mastectomy. Surg Clin North Am 1983;63(6): I33 |-489.

8. Gaskin TA, Lobuglio A, Kelly P, Doss M, Pizitz N. Stretch: a rehabilitive program for patients with breast cancer. South Med J 1989;82:467-9.

9. Chen SH, Chen MF. Timing of shoulder exercise after modified radical mastectomy: a prospective study. Chang Gung Med J 1999;22: 37-43.

10. Molinaro J, Kleinfeld M, Lebed S. Physical therapy and dance in the surgical management of breast cancer. Phys Ther 1986,66:967-9.

II. Silva MPP, Derchain SFM, Rezende L, Cabello C, Martinez EZ. Movimento do ombro após cirurgia por carcinoma invasor da mama: estudo randomizado prospectivo controlado de exercícios livres versus limitados a $90^{\circ}$ no pós-operatório. Rev Bras Ginecol Obstet 2004;26: I 25-30.

12. Erickson VS, Pearson ML, Ganz PA, Adams J, Kahn KL. Arm Edema in Breast Cancer Patients. J Natl Cancer Inst 200 I;97:96- I I I.

13. Declaração de Helsinque III Sobre os princípios éticos para pesquisas em seres humanos [citado I5 nov 2003]. Disponível em: http:// www.ibemol.com.br/declarações/helsinque 3.asp.

14. Brasil. Ministério Da Saúde. Conselho Nacional da Saúde. Resolução CNSI 96/96 sobre pesquisa envolvendo seres humanos. Inf Epidemiol SUS 1996;2.

15. Flew TJ. Wound drainage following radical mastectomy: the effect os restriction of shoulder movement. Br J Surg 1979;66:302-5.

16. Dawson I, Stam L, Heslinga JM, Kalsbeek HL. Effect of shoulder immobilization on wound seroma and shoulder dysfuntion following modified radical mastectomy: a randomized prospective clinical trial. $\mathrm{Br}$ ] Surg 1990;76:3।1-2.

17. Jansen RFM, Van Geel AN, De Groot HGW, Rottier AB, Olthuis GAA, et al. Immediate versus delayed shoulder exercises after axillary lymph node dissection. Am J Surg 1990; 1 60:481-4.

18. Knight CD Jr, Griffen FD, Knight SCD. Prevention of seroma in mastectomy wounds. The effect of shoulder immobilization. Arch Surg 1995: |30:99-|0|.

19. Bland K, Copeland EM. A mama. São Paulo: Manole; 1994.

Artigo recebido: 21/09/04

Aceito para publicação: 16/09/05 